\title{
Avaliação comparativa entre maturadores de cana de açúcar (Saccharum officinarum $L$.) na região Oeste de São Paulo
}

Comparative evaluation between sugarcane (Saccharum officinarum L.) ripeners in the western region of São Paulo

Evaluación comparativa entre maduradores de caña de azúcar (Saccharum officinarum

L.) en la región Oeste de São Paulo

Fernando Takayuki Nakayama Pesquisador científico Doutor, APTA-PR Alta Paulista, Brasil fnakayama@apta.sp.gov.br

Samuel Ferrari Professor Doutor, Unesp-Dracena, Brasil. ferrari@dracena.unesp.br

Raquel Nakazato Pinotti Pesquisador Científico Doutor, APTA Regional-Bauru/SAA-SP, Brasil raquelnakazato@apta.sp.gov.br 


\section{RESUMO}

O presente trabalho teve por um objetivo comparar o efeito de diferentes maturadores, sobre os seus ganhos nos teores de ATR na cana-de-açúcar. O experimento foi conduzido em cana soca de segundo corte, variedades SP891115, RB85-5453 e CTC4 na Fazenda Coroados, localizada no município de Nova Independência, estado de São Paulo, durante ano de 2014. O delineamento experimental utilizado foi o de blocos casualizados com seis repetições. Os tratamentos consistiram da aplicação dos seguintes produtos: Sulfometuron metil 0,02 $\mathrm{Kg} / \mathrm{ha}$, Bispyribac-sodium 0,075 L/ha ,Orthosulfamuron 0,150 L/ha, Cletodin 0,150 L/ha e a testemunha (maturação natural). As avaliações foram realizadas no intervalo de 5 dias até a colheita. Na comparação entre os produtos Sulfometuron metil e Cletodin não foram encontradas diferenças entre os produtos. Já no segundo experimento o maturador Orthosulfamuron apresentou ganhos de ATR inferior ao Sulfometuron metil diferindo significativamente deste; Na terceira experimentação, quando o Sulfometuron metil foi comparado com o Bispyribac-sodium, concluise que o segundo contribuiu para a melhoria da qualidade tecnológica da cana de açúcar, proporcionando resultados semelhantes aos observados nos tratamentos com Sulfometuron metil.

PALAVRAS-CHAVE: Maturadores. Qualidade da cana de açúcar. ATR (açúcar total recuperável)

Abstract. The objective of the present work was to compare the effect of different ripeners on their gains in ATR levels in sugarcane. The experiment was carried out in second - crop soca cane, varieties SP89-1115, RB85-5453 and CTC4 at Coroados farm, located in Nova Independência-SP, during the year 2014. The experimental design used was block with six repetitions. The treatments consisted of the application of the following products: Sulfometuron methyl $0.02 \mathrm{~kg} / \mathrm{ha}$, Bispyribac-sodium 0,075 L / ha, Orthosulfamuron 0,150 L / ha, Cletodin 0,150 L / ha and the control (natural maturation). The evaluations were carried out in the interval of 5 days until harvest. In the comparison between Sulfometuron methyl and Cletodin products, no differences were found between products. In the second experiment, the Orthosulfamuron maturation group presented lower ATR gains than Sulfometuron methyl differing significantly from this; In the third experiment, when Sulfometuron methyl was compared with Bispyribac-sodium, it was concluded that the second one contributed to the improvement of the technological quality of sugarcane, providing results similar to those observed in Sulfometuron methyl treatments.

Keywords: Ripeners. Quality sugarcane. ATR (fully recoverable sugar)

Resumen. El presente trabajo tuvo como objetivo comparar el efecto de diferentes maduradores, sobre sus ganancias en los niveles de ATR en la caña de azúcar. El experimento fue conducido en caña soca de segundo corte, var. SP89-1115, RB85-5453 y CTC4 en la Hacienda Coroados, ubicada en el municipio de Nova Independencia-SP, durante el año 2014. El delineamiento experimental utilizado fue el de bloques con las seis repeticiones. Los tratamientos consistieron en la aplicación de los siguientes productos: Sulfometuron metil 0,02 Kg / ha, Bispyribacsodium 0,075 L / ha, Orthosulfamuron 0,150 L / ha, Cletodin 0,150 L / ha y el testigo (maduración natural). Las evaluaciones se realizaron en el intervalo de 5 días hasta la cosecha. En la comparación entre los productos Sulfometuron metil y Cletodin no se encontraron diferencias entre los productos. Ya en el segundo experimento el matador Orthosulfamuron presentó aumentos de ATR inferior al Sulfometuron metil diferenciando significativamente de éste; En la tercera experimentación, cuando el Sulfometuron metil se comparó con el Bispyribac-sodium, se concluyó que el segundo contribuyó a la mejora de la calidad tecnológica de la caña de azúcar, proporcionando resultados similares a los observados en los tratamientos con Sulfometuron metil.

Palabras clave: Madurador. Calidad del caña de azúcar de. ATR (azúcar totalmente recuperable) 


\section{Introdução}

A cana de açúcar Saccharum officinarum L. é considerada uma planta de múltipla utilidade, podendo ser empregada in natura, sob a forma de forragem, para alimentação animal, ou como matéria prima para a fabricação de rapadura, melado, aguardente, açúcar e álcool. Seus resíduos têm grande importância econômica: o vinhoto é transformado em fertilizante e o bagaço na forma de biomassa, em combustível. O Brasil lidera a lista dos 80 países produtores, respondendo por 25\% da produção mundial. Na safra de 2017/2018 o Brasil obteve a produção de 633,26 milhões de toneladas de cana-de-açúcar (CONAB, 2018). No Brasil, o complexo sucroalcooleiro gera uma renda de US\$ 7 bilhões, sendo que US\$ 3,2 bilhões são obtidos em vendas para o exterior. A cana-de-açúcar é a base para todo o agronegócio sucroalcooleiro, representado por 350 indústrias de açúcar e álcool e 1.000 .000 empregos diretos e indiretos. O cultivo da cana-de-açúcar tem se expandido para o Oeste Paulista que tem grande importância nesta expansão. Tradicional na agropecuária, a região apresenta importância também no cultivo de cana-de-açúcar, por suas características físicas, com disponibilidade de terra, topografia plana que propicia a mecanização da colheita, clima favorável apresentado verão chuvoso e inverno seco, logística em pleno desenvolvimento com multimodais para o escoamento da produção, dentre outros fatores, como a saturação da região tradicional.

Um dos aspectos relevantes que pode ser melhorado para prolongar o período de safra é melhorar os teores de açúcar nos períodos chuvosos, que naturalmente caracteriza-se por apresentar a planta vegetando, antecedendo o período de maturação que acumula açúcares. O processo de maturação da cana-de-açúcar pode ser definido como o processo fisiológico que envolve a formação de açúcares nas folhas e armazenamento no colmo. Desta forma, para a maximização e melhoria do aspecto econômico, é compensatório que a agroindústria aumente o período de safra, que na região do Oeste Paulista limita-se pelo período chuvoso e teor de açúcar na planta. Para tal, é cada vez racional a utilização de ferramentas que melhorem as características de qualidade tecnológica da cana, destacado pelo acúmulo de açúcares (Caputo, 2007).

Os maturadores, definidos como reguladores vegetais, agem alterando a morfologia e a fisiologia da planta, podendo-se levar a modificações qualitativas e quantitativas na produção. Podem atuar promovendo a diminuição do crescimento da planta, possibilitando incrementos no teor de sacarose, precocidade de maturação, aumento de produtividade, e também atuar sobre as enzimas (invertases), que catalisam acúmulo de sacarose nos colmos. Sua aplicação no sistema de produção de cana-de-açúcar tem proporcionado uma maior flexibilidade no gerenciamento da colheita, altamente relevante para o planejamento da produtividade da cultura, além de propiciar à industrialização de uma matéria-prima de melhor qualidade.

Segundo Taupier (1999), um hectare de cana-de-açúcar é capaz de produzir 100 toneladas de matéria verde a cada ano, superando duas vezes o rendimento agrícola de outras culturas energéticas; contribui com o equivalente a 10 toneladas de petróleo. 0 caldo extraído (caldo mesclado) tem por volta de $15^{\circ}$ Brix com uma pureza que flutua entre 80 e $87 \%$. Portanto, 100 $\mathrm{t}$ de caldo devem conter $15 \mathrm{t}$ de sólidos totais e entre 12 e $13 \%$ de Pol. 
Os maturadores, definidos como reguladores vegetais, agem alterando a morfologia e a fisiologia da planta, podendo levar a modificações qualitativas e quantitativas na produção. A utilização dos reguladores vegetais poderá ser uma alternativa compensadora aos investimentos e objetivos propostos à cultura da cana, por possibilitarem incrementos no teor de sacarose, precocidade de maturação e aumento na produtividade (Martins \& Castro, 1999). A aplicação de maturadores vegetais na cultura da cana-de-açúcar tem se tornado, prática cada vez mais comum no setor sucroalcooleiro, sobretudo em regiões que caracterizam-se por limitar a janela de safra da cana com períodos chuvosos que atrasam o início da safra, como é o caso do Oeste Paulista. O objetivo é antecipar e manter a maturação natural e assim disponibilizar matéria-prima de boa qualidade para industrialização antecipada, além de auxiliar no manejo das variedades (Gheller, 2001).

\section{Objetivo}

O objetivo do presente trabalho foi avaliar diferentes princípios ativos de maturadores de cana-de-açúcar e respectivas dosagens em canaviais do oeste paulista.

\section{Material e Métodos}

Foram instalados 3 experimentos com testes comparativos localizadas na mesma propriedade rural, sendo conduzidos em cana soca, respectivamente com as variedades SP89-1115, RB855453 e CTC4, em seu $2^{\circ}$ corte na Fazenda Coroados com coordenadas $21^{\circ} 07^{\prime} 10^{\prime \prime}$ S e $51^{\circ} 25^{\prime} 0^{\prime \prime} \mathrm{O}$, localizada no município de Nova Independência, Estado de São Paulo (figura 02), pertencente ao Grupo Pedra Agroindustrial - Unidade Ipê 


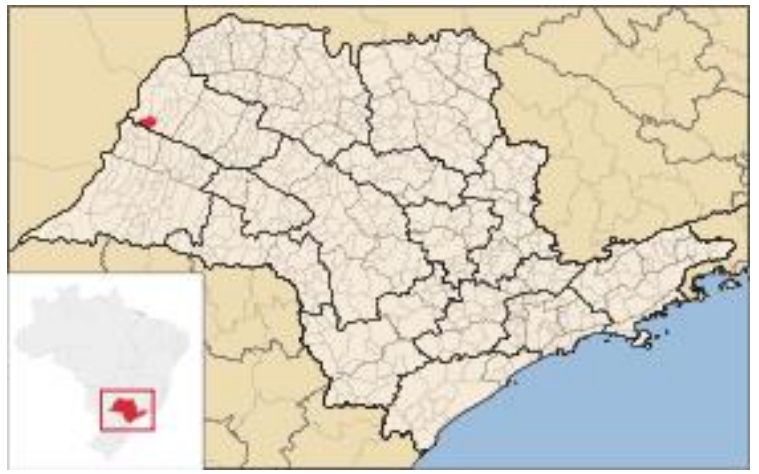

Figura 02.

Fonte: Wikipédia (2014)

O período da experimentação correspondeu aos meses de Abril e Maio de 2014, onde realizaram-se as aplicações dos produtos por meio de aplicação aérea sendo o volume de calda de 30 litro/ha.

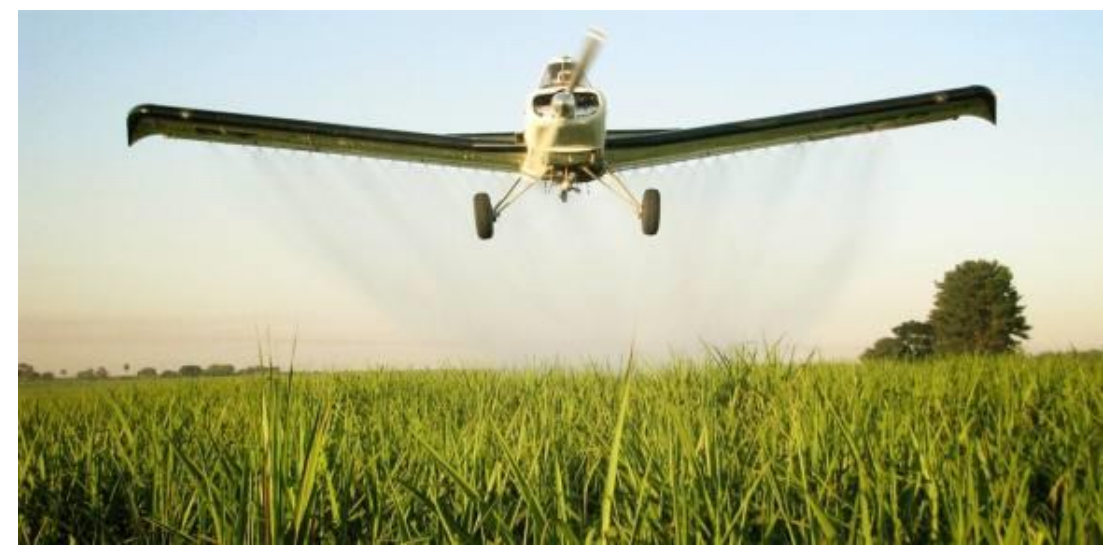

Figura 03

Fonte: Usina Ipê

Para a preparação das áreas experimentais, foram utilizadas lonas plásticas de $16 \mathrm{~m}^{2}$ em 6 repetições, das quais cobriram a área destinada para o tratamento testemunha, isentando-a da susceptibilidade da recepção de produtos, como demonstrado na (figura 1). Desta forma foram colhidas posteriormente amostras da área aplicada (tratado) e não aplicada (testemunha protegidas por lonas). 


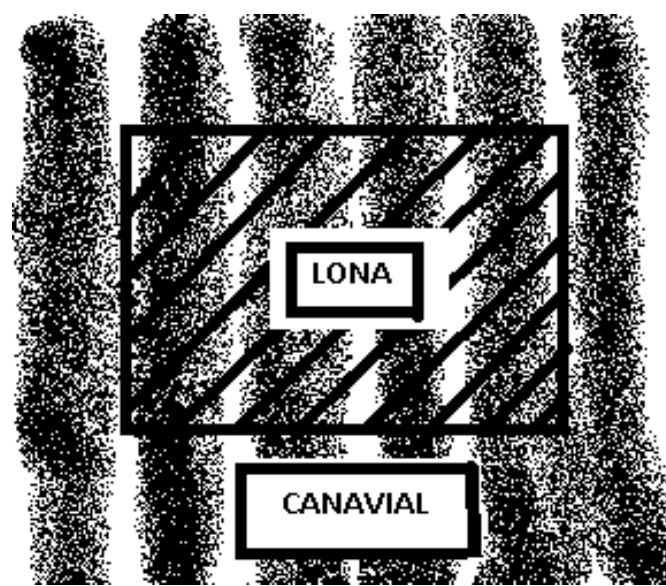

Figura 04

Cada amostra é composta de $1,0 \mathrm{~m}^{2}$ de cana, onde foram retiradas todas as canas industrializáveis, pois serão 12 amostras por tratamento, sendo 6 no local onde foi colocado a Iona e 6 fora da lona que foi aplicado produto: Portanto retirar as amostras um dia antes da aplicação e depois espaçadas de 5 dias até a colheita, sempre retirando as amostras nos locais próximos a primeira amostragem, os colmos coletados encaminhados para o laboratório da usina para serem processados, segundo metodologia do Sistema de Pagamento de Cana pelo Teor de Sacarose, descritas em Fernandes (2003), onde foram considerados os parâmetros: açúcar total recuperável (ATR), sólidos solúveis (Brix em \%), sacarose (Pol do caldo em \%), fibra industrial na cana (\%) e produtividade (t/ha), tendo como ênfase este experimento a comparação dos teores de ATR de acordo com princípios ativos diferentes.

Tratamentos

Experimento 01:

Os tratamentos do experimento 01 consistiram na utilização de 2 princípios ativos registrados comercialmente para a cultura de cana de açúcar comparados à testemunha. Foram utilizadas as dosagens recomendadas pelo fabricante de cada produto. Segue abaixo os produtos e doses aplicados:

1- Testemunha;

2- SULFOMETURON METIL 0,02 Kg/ha;

3- CLETODIN 0,150 L/ha.

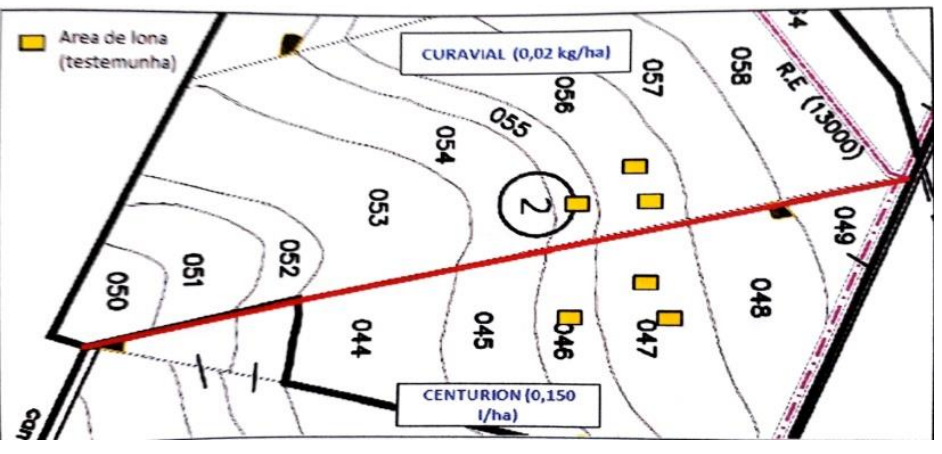

Figura 05

Experimento 02: 
Os tratamentos do experimento 03 consistiram na utilização de 2 principios ativos registrados comercialmente para a cultura de cana de açúcar comparados à testemunha. Foram utilizadas as dosagens recomendadas pelo fabricante de cada produto. Segue abaixo os produtos e doses aplicados:

1- Testemunha;

2- SULFOMETURON METIL 0,02 Kg/ha;

3- ORTHOSULFAMURON 0,150 L/ha

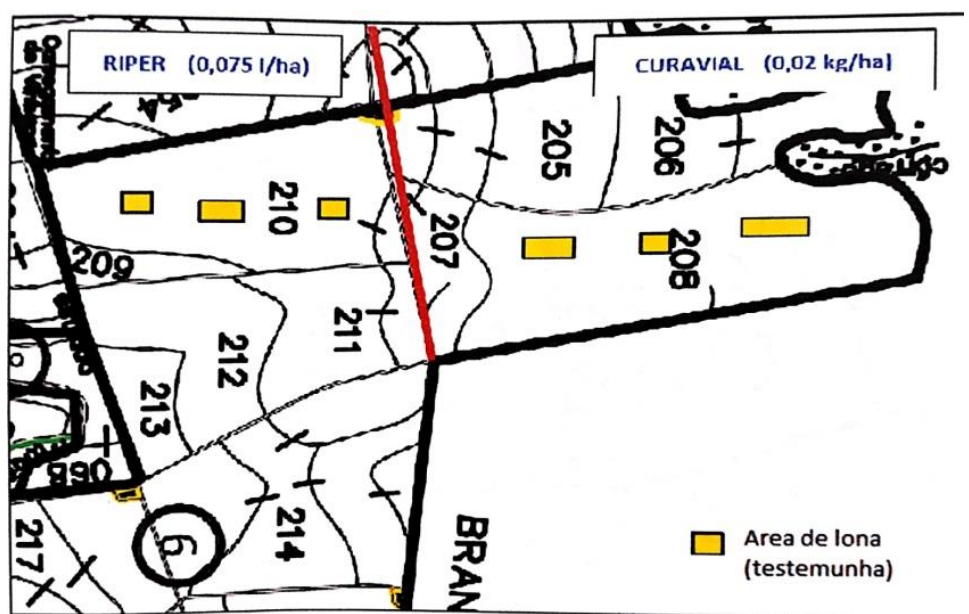

Figura 06

Experimento 03:

Os tratamentos do experimento 02 consistiram na utilização de 2 princípios ativos registrados comercialmente para a cultura de cana de açúcar comparados à testemunha. Foram utilizadas as dosagens recomendadas pelo fabricante de cada produto. Segue abaixo os produtos e doses aplicados:

1- Testemunha;

2- SULFOMETURON METIL 0,02 Kg/ha;

3- BISPYRIBAC-SODIUM 0,075 L/ha;

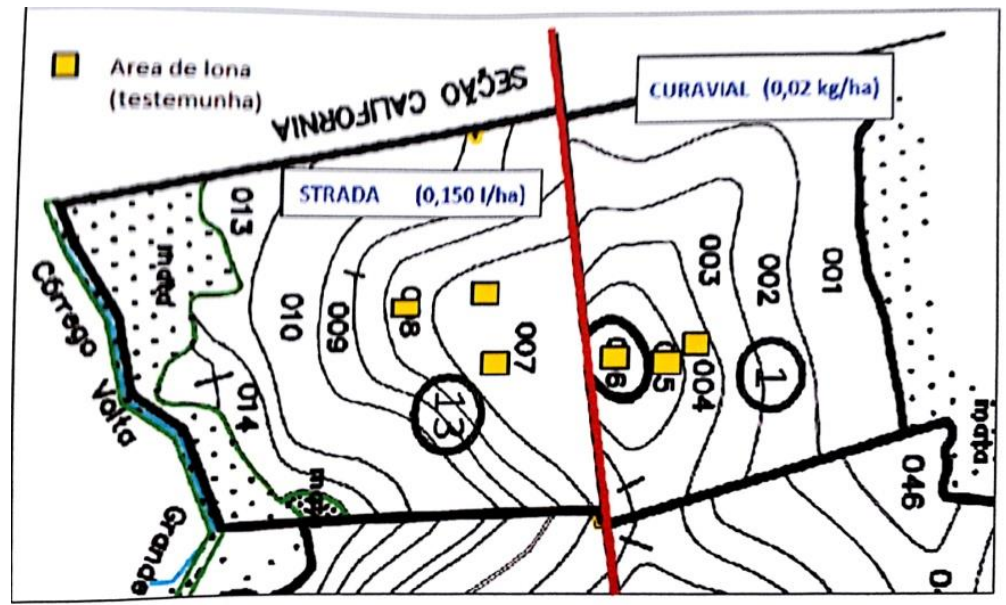

Figura 07 
Os resultados obtidos foram submetidos à análise de variância pelo teste $\mathrm{F}$ e as médias foram comparadas pelo de Tukey, ao nível de $5 \%$ de probabilidade, utilizando o software estatístico ASSISTAT.

\section{Resultados e Discussão}

Na safra 2014, os regimes hídricos e térmicos (CIIAGRO, 2014) não favoreceram o desenvolvimento vegetativo na fase inicial da cana-de-açúcar, tendo em vista a irregular distribuição da precipitação no decorrer da fase vegetativa. No mês de abril de 2014 a precipitação $(80 \mathrm{~mm})$ favoreceu a retomada de vegetação contudo manteve os índices de açúcar em níveis abaixo do ideal para a colheita. Neste sentido, a utilização de maturadores vem como uma importante ferramenta, sendo indicada em casos de planejamento de colheita auxiliando no acúmulo e/ou concentração antecipada de ATR. No experimento 01 instalado na fazenda Coroados, aplicou-se os produtos Sulfometuron Metil e Cletodin em talhão previamente demarcado e delineado para posterior verificação de efeito maturador. 0 produto Sulfometuron Metil é considerado como padrão de maturador dentro da empresa, sendo que o principal intuito deste experimento é compará-lo com Cletodin bem como a testemunha. Nas 5 avaliações realizadas, não foram encontradas diferenças estatísticas, porém identificou-se valores superiores nas áreas tratadas em relação a testemunha. Apesar de não diferir estatisticamente pelo teste de Tukey, nas avaliações de 19/04 e 26/04 identificaram-se valores de 15,95 e 26,01 kg.ton ${ }^{-1}$. Destacando que a média de produtividade para safra 2014 da fazenda Coroados era por volta de 75 ton $^{-1}$ pode-se destacar que a aplicação de maturador poderia acrescentar $1950 \mathrm{~kg}$ de açúcar por ha. Neste sentido, considerando que a cotação de ATR em 30 de abril era de $R \$ 0,48 . \mathrm{kg}^{-1}$, possivelmente poderia acrescentar a receita valores em torno de $\mathrm{R} \$ 936,00$ por ha com a utilização de maturadores.

Tabela 01. Valores médios de ATR $\left(\mathrm{kg}^{\mathrm{t}} \mathrm{ton}^{-1}\right.$ ), obtidos em cana de açúcar (Saccharum officinarum) var. SP 89-1115 submetidos à aplicação de maturadores. Nova Independência, SP. 2014.

\begin{tabular}{llllll}
\hline Avaliações & $05 / 04$ & $10 / 04$ & $15 / 04$ & $19 / 04$ & $26 / 04$ \\
\hline Teste F & $1,5529 . n . s$ & $2,0689 . n . s$ & $0,2984 . n . s$ & $0,6959 . n . s$ & $2,4451 . n . s$ \\
C.V.\% & 8,67 & 11,99 & 13,79 & 16,38 & 16.00 \\
D.M.S. & 19,12 & 23,88 & 31,81 & 41,68 & 39,72 \\
\hline \multicolumn{5}{c}{ Maturadores } \\
\hline Testemunha & 82,98 & 79,68 & 87,50 & 93,10 & 82,85 \\
SULFOMETURON METIL & 87,30 & 71,50 & 94,95 & 102,43 & 105.72 \\
CLETODIN & 93,82 & 87,33 & 93,79 & 109,05 & 108,86 \\
\hline
\end{tabular}

As médias seguidas pela mesma letra minúscula na coluna diferem estatisticamente entre si. Foi aplicado o Teste de Tukey ao nível de 5\% de probabilidade.

No experimento 02, também instalado na Fazenda Coroados município de Nova Independência-SP aplicaram-se os produtos Sulfometuron Metil e Orthosulfamuron em talhão previamente demarcado e delineado para posterior verificação do efeito maturador (figura 
06). O produto Sulfometuron Metil é considerado como padrão de maturador pela usina, sendo que o principal intuito deste experimento é compará-lo com Orthosulfamuron e com o tratamento testemunha. Dentre 4 avaliações realizadas, somente na avaliação do dia 03/04 foram encontradas diferenças pelo teste de Tukey a $5 \%$ de probabilidade, no qual resultou um ganho de ATR de 16,35 kg.ton ${ }^{-1}$ sendo que o produto Sulfometuron Metil superou em ATR o maturador Orthosulfamuron. MESCHEDE (2009) estudando Sulfometuron Metil juntamente com Glyfosate obteve ganho de $14 \%$ de ATR em relação a testemunha, destacando a eficiência deste produto e concordando com os acréscimos deste estudo. Considerando que a média de safra 2014 da fazenda Coroados situa-se por volta de 75 ton. ha ${ }^{-1}$ salienta-se que poderia haver acréscimos de 1226,25 kg de açúcar por ha. Neste sentido, considerando a cotação de ATR na época era de $\mathrm{R} \$ 0,48 \mathrm{~kg}^{-1}$, possivelmente poderia acrescentar à receita valores em torno de $\mathrm{R} \$ 588,60$ por ha com a utilização do Sulfometuron Metil.

Tabela 02. Valores médios de ATR $\left(\mathrm{kg} \cdot \mathrm{ton}^{-1}\right)$, obtidos em cana de açúcar (Saccharum officinarum) var. RB85-5453 submetidos à aplicação de maturadores. Nova Independência, SP. 2014.

\begin{tabular}{lllll}
\hline Avaliações & $03 / 04$ & $12 / 04$ & $19 / 04$ & $26 / 04$ \\
\hline Teste F & $0,048451^{*}$ & $2,2281 . n . s$ & $2,4326 . n . s$ & $1,8147 . n . s$ \\
CV(\%) & 7,77 & 10,23 & 10,46 & 8,22 \\
DMS & 13,76 & 15,51 & 16,54 & 13,78 \\
\hline \multicolumn{5}{c}{ Maturadores } \\
\hline Testemunha & $119,64 \mathrm{ab}$ & 103,78 & 110,78 & 114,29 \\
SULFOMETURON METIL & $125,80 \mathrm{a}$ & 105,87 & 108.24 & 115,44 \\
ORTHOSULFAMURON & $109,45 \mathrm{~b}$ & 94,05 & 97.54 & 106.15 \\
\hline
\end{tabular}

As médias seguidas pela mesma letra minúscula na coluna diferem estatisticamente entre si. Foi aplicado o Teste de Tukey ao nível de $5 \%$ de probabilidade.

No experimento 03, também instalado na mesma localidade porém em outro talhão, foram comparados os produtos Sulfometuron Metil e Bispyribac-sodium em talhão previamente demarcado e delineado para posterior verificação de diferenças. Conciani et al. (2012), em trabalho semelhante, conclui que o Bispyribac-sodium obteve resultados significativos em relação a testemunha, contribuindo para a melhoria da qualidade tecnológica da cana-deaçúcar, proporcionando resultados semelhantes aos observados nos tratamentos com Sulfometuron metil. Estes resultados corroboram com o presente estudo pois dentre as 4 avaliações realizadas após a aplicação, foram encontradas diferenças estatísticas em duas datas: 12/05 e 26/05 pelo teste de Tukey, no qual resultou um ganho de ATR de 4,51 e 0,92 $\mathrm{kg} \mathrm{ton}^{-1}$ respectivamente do produto Bispyribac-sodium em relação ao Sulfometuron metil, porém diferindo estatisticamente somente do tratamento testemunha. No mesmo raciocínio dos experimentos anteriores, considerando que a média de safra 2014 da fazenda Coroados situa-se por volta de 75 ton. ha ${ }^{-1}$ poderia ser encontrados acréscimos de 338,25 kg de açúcar por ha. Neste sentido destacando a cotação de ATR em 30 de maior era R\$ 0,46. $\mathrm{kg}^{-1}$, possivelmente acrescentaria valores em torno de $R \$ 155,00$ por ha com a utilização de Bispyribac-sodium. 
Tabela 03. Valores médios de ATR $\left(\mathrm{kg}^{\mathrm{t}} \mathrm{ton}^{-1}\right.$ ), obtidos em cana de açúcar (Saccharum officinarum) var. CTC 04 submetidos à aplicação de maturadores. Nova Independência, SP. 2014.

As

\begin{tabular}{lllll}
\hline Avaliações & $03 / 05$ & $12 / 05$ & $19 / 05$ & $26 / 05$ \\
\hline Teste F & $0,6237 . n . s$ & $0,037848^{*}$ & $0,4743 . n . s$ & $0,044199^{*}$ \\
C.V.\% & 6,87 & 6,08 & 6,07 & 5,25 \\
D.M.S. & 10,99 & 10,53 & 10,92 & 9,85 \\
\hline \multicolumn{5}{c}{ Maturadores } \\
\hline Testemunha & 106,47 & $109,71 \mathrm{~b}$ & 119,72 & $118,89 \mathrm{~b}$ \\
SULFOMETURON METIL & 104,66 & $116,30 \mathrm{ab}$ & 118,14 & $128,18 \mathrm{ab}$ \\
BISPYRIBAC-SODIUM & 109,35 & $120,81 \mathrm{a}$ & 122,20 & $129,10 \mathrm{a}$ \\
\hline
\end{tabular}

médias seguidas pela mesma letra minúscula na coluna diferem estatisticamente entre si. Foi aplicado o Teste de Tukey ao nível de 5\% de probabilidade.

\section{Conclusão}

$\mathrm{Na}$ comparação entre os produtos Sulfometuron metil e Cletodin não foram encontradas diferenças entre os produtos. Já no segundo experimento o maturador Orthosulfamuron apresentou ganhos de ATR inferior ao Sulfometuron metil diferindo significativamente deste; $\mathrm{Na}$ terceira experimentação, quando o Sulfometuron metil foi comparado com o Bispyribacsodium, conclui-se que o segundo contribuiu para a melhoria da qualidade tecnológica da cana de açúcar, proporcionando resultados semelhantes aos observados nos tratamentos com Sulfometuron metil. Destaca-se que no presente estudo foram encontrados ganhos em 3 variedades de cana de açúcar, o que comprova a não seletividade varietal diante da utilização de maturadores. Desta forma podemos salientar que além do produto padrão, sendo atualmente o mais utilizado pelas usinas da região, existem opções com eficiência semelhantes, ficando a critério do usuário a escolha da melhor opção.

\section{REFERÊNCIAS BIBLIOGRÁFICAS}

CAPUTO, M. M. et al. Acúmulo de sacarose, produtividade e florescimento de cana-de-açúcar sob reguladores vegetais. Asociación Interciencia, v. 32, n. 12, Caracas, Dez., 2007.

CIIAGRO - Centro Integrado de Informações Agrometeorológicas. CIIAGRO Online [base de dados na internet]. São Paulo: Instituto Agronômico [s.d.] [acesso em 14 jun 2014]. Disponível em: http://www.ciiagro.sp.gov.br/ciiagroonline/ Links ]ciiagro.sp.gov.br/ciiagroonline/"> .

COMPANHIA NACIONAL DE ABASTECIMENTO - CONAB. 2018. Disponível em: https://www.conab.gov.br/ultimas-noticias/2327-cana-de-acucar-tem-queda-de-3-6-e-fecha-safra2017-18-em-633-26-milhoes-de-t Acesso em: 14 jun 2018. 
CONCIANI P.A. INOUE M.H.; CAPPELLESSO E.J.S.; BEM R.; PEREIRA R.L.; BICA A.L. Eficiência do Riper (Bispyribac-sodium) como maturador na cultura da cana de açúcar. li. Características Tecnológicas. In: XXVIII Congresso Brasileiro de Ciencia das Plantas daninhas na era da biotecnologia, Aárea 2-Fisilogia e metabolismo de herbicidas, Campo Grande. Anais... XXVIII CBCPD, 3 a 6 de setembro de 2012, Campo Grande MS. 2012.

EMPRESA BRASILEIRA DE PESQUISA AGROPECUÁRIA - EMBRAPA. 2011. Agencia Emprapa de informação tecnológica. Disponível em: HTTP://www.agencia.cnptia.embrapa.br/gestor/cana-deacucar/arvore/CONTAG01 33 711200516717.html Acesso em: 12 jun 2018

FERNANDES, A.C. Calculos na agroindústria da cana-de-acucar. 2. Piracicaba: EME, 2003. 240p.

GHELLER, A. C. A. Resultados da aplicação de maturadores vegetais em cana-de-açúcar, variedades RB72454 e RB835486 na região de Araras, SP. In: 4 Jornada Científica da UFSCar, 2001, São Carlos. Resumos... 2001.

MESCHEDE D.K.; Efeito do glyphosate e sulfumeturon-metil na fisiologia da cana-de-acucar. 2009. $77 f$. tese (doutorado) - Universidade Estadual Paulista - Faculdades de ciências agrárias de Botucatu, Botucatu, 2009.

RODRIGUES, J.D. Fisiologia da cana-de-açúcar. Botucatu: Instituto de Biociências -Universidade Estadual Paulista, 1995. 99p.

TAUPIER, L. O. et al. A cana-de-açúcar. In:ICIDCA. Manual dos Derivados da Cana-de-Açúcar: diversificação, matérias-primas, derivados do bagaço, derivados do melaço,outros derivados, resíduos, energia. Brasília:ABIPTI, 1999. Cap. 2.1, p.21-27.

WIKIPÉDIA. Desenvolvido pela Wikimedia Foundation. Apresenta conteúdo enciclopédico. Disponível em: https://pt.wikipedia.org/wiki/Nova Independ\%C3\%AAncia. Acesso em 21 jun 2014. 